\title{
Instrumentation légère pour la mesure de l'érodabilité des sédiments vaseux ou sablo-vaseux
}

\author{
Pierre Le Hir, Philippe Cann, Hervé Jestin et Philippe Bassoullet \\ IFREMER, centre de Brest, BP 70, 29280 Plouzané
}

\begin{abstract}
Résumé
L'érodimètre est un instrument portable destiné à déterminer les conditions d'érosion (seuil éventuel et flux d'érosion) d'un sédiment fin, vaseux ou sablovaseux. Le principe consiste à soumettre un échantillon de sédiment non remanié à un courant forcé dans un canal. La remise en suspension de particules fines est contrôlée en continu par un turbidimètre, tandis que les sables sont récupérés dans un piège. Exprimé en contrainte de cisaillement, le forçage peut atteindre $30 \mathrm{~Pa}$, et a été calibré jusqu'à une valeur de $12 \mathrm{~Pa}$. Tandis que la pertinence d'un seuil d'érosion ainsi que la représentativité des mesures sont abordés, des premiers résultats sont donnés: l'instrument permet en particulier de caractériser l'érosion des mélanges naturels de sable et de vase.
\end{abstract}

\begin{abstract}
The erodimetre is a portable device dedicated to the characterisation of fine sediments erodability (erosion flux and possible threshold). The principle consists in generating a current-induced shear stress on a sample of undisturbed sediment, in a small flume. Resuspension of fine particles is controlled by a turbidimetre, whereas sands in motion are collected in a trap downstream of the sediment. The shear stress can reach $30 \mathrm{~Pa}$, and has been calibrated up to $12 \mathrm{~Pa}$. The relevance of the erosion threshold and the natural variability of measurements are slightly discussed. First results are given: the instrument can be used to characterize the erosion of sand and mud mixtures.
\end{abstract}

Mots clés: contrainte de cisaillement, érosion, sédiments cohésifs, mélanges sable/vase

\section{Introduction}

La vitesse de chute et l'érosion des sédiments sont les principaux processus qui contrôlent les mouvements de sédiments fins. En particulier dans le cas des sédiments cohésifs, les processus de tassement sont susceptibles de faire évoluer au cours du temps l'érodabilité d'un sédiment, c'est-à-dire sa capacité à être mis en mouvement ou en suspension sous l'action d'un forçage hydrodynamique. Classiquement, ce forçage est paramétré par la contrainte de cisaillement au niveau de l'interface eau/sédiment, encore appelée "contrainte de peau". L'érodabilité est a priori caractérisée par un flux d'érosion (en masse ou volume 
par unité de temps et de surface) que l'on cherche à exprimer en fonction de la contrainte hydrodynamique (qu'elle soit liée à un courant ou des vagues) et des caractéristiques du sédiment. Le cas échéant, un seuil d'érosion peut apparaître, il représente alors la valeur critique de la contrainte de cisaillement en dessous de laquelle il n'y a pas d'entraînement du sédiment par le fluide .

Si les conditions de remaniement des sables sont relativement bien connues depuis les travaux de Shields, il n'en n'est pas de même des sédiments cohésifs pour lesquels il n'existe pas de loi d'érosion universelle (re-)connue, et l'on doit encore réaliser des tests pour caractériser leur érodabilité. Il s'agit le plus souvent d'expériences dans des canaux de laboratoire sur des sédiments remaniés (e.g. Migniot, 1989). Pour éviter certains artefacts, des instruments de mesure in situ ont été développés depuis une vingtaine d'année (Black et al., 1997; Tolhurst et al., 2000). Mais il ne s'agit encore que de prototypes : lorsque leur mise en œuvre est en pleine eau, elle est délicate et longue, et lorsque les instruments sont déployés sur estran, des doutes subsistent sur la similitude de comportement du sédiment lorsqu'il est recouvert d'eau ou non. Enfin, il faut relever que si de nombreuses séries de tests ont été réalisées sur des vases, peu l'ont été sur des sédiments mixtes (mélanges sable/vase), qui ont souvent un comportement cohésif et qui sont extrêmement répandus dans les baies semi-fermées ou sur le plateau continental, à des profondeurs où l'action des houles ou du courant sont atténuées.

Avec le soutien du Programme National d'Environnement Côtier, IFREMER a développé l'"érodimètre", un instrument adapté à la mesure de l'érodabilité de sédiments peu ou pas remaniés, vaseux ou sablo-vaseux. Le parti de permettre des tests de courte durée et de pouvoir les reproduire fréquemment a été pris, de façon à obtenir une information statistique sur l'érodabilité des sédiments naturels, dont on connaît la variabilité. De ce fait, il a été admis que la surface de sédiment testée pouvait être réduite.

\section{Description de l'érodimètre}

\subsection{Principe de mesure}

Une première version de l'érodimètre avait pour principe de générer un écoulement dans une cellule coiffant un échantillon de sédiment (Paillard et al., 1998). Des difficultés d'échantillonnage de sédiment non remanié sur une longueur suffisante, et la variabilité de la couche limite le long du sédiment ont conduit à allonger le canal tout en réduisant l'interface avec le sédiment.

Le principe adopté est de faire affleurer l'échantillon de sédiment à tester à la base d'un petit canal dans lequel un écoulement en circuit fermé est forcé par une pompe contrôlée par un variateur de fréquence. L'écoulement est incrémenté par paliers, et pour chacun d'eux la quantité de matières en suspension (M.E.S.) est mesurée à l'aide d'un turbidimètre à rétrodiffusion optique (de type Sea Point). L'homogénéité du mélange est rapidement établie dans le circuit. En outre, un 
piège à sable localisé en aval de l'échantillon de sédiment permet de récupérer le sable mis en mouvement mais non transporté en suspension dans le système. Le volume récupéré est noté à la fin de chaque palier, tandis qu'en fin de test la masse totale de sable est pesée.

L'échantillon est délicatement transféré dans le porte-échantillon depuis la carotte de prélèvement, à l'aide d'un piston adapté à la carotte. On vérifie que le sédiment ne subit pas d'altération pendant l'opération. Sur estran découvert, le sédiment est prélevé à la main dans une carotte courte (30 $\mathrm{cm}$ environ), tandis qu'en zone subtidale, un carottier préservant l'interface est utilisé: c'est un multitubes de Bowers \& Connelly. Pour une mesure, environ $5 \mathrm{~cm}$ de sédiment sont mis en place dans le porte-échantillon, et la surface du sédiment restant est arasée, de façon à permettre un autre test sur le sédiment encore dans la carotte. Cette approche permet d'obtenir un profil vertical discret de l'érodabilité du sédiment. Un certain nombre de mesures ont aussi été faites sur des sédiments préparés en laboratoire, directement placés dans le porte-échantillon.

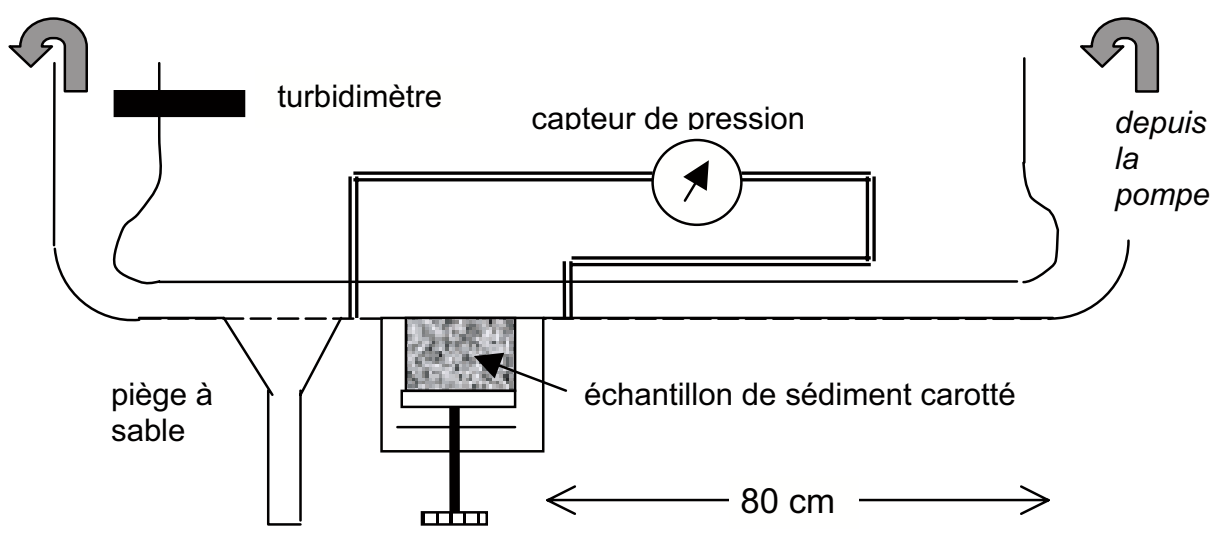

Figure 1 Principe de fonctionnement de l'érodimètre

Après érosion du sédiment, qui n'est jamais prolongée de façon à ne pas modifier la hauteur d'écoulement (et donc la vitesse) de plus de $10 \%$, des prélèvements sont effectués pour des mesures de concentration/teneur en eau et de microgranulométrie (au laser). Une photo de la surface de sédiment est prise avant et après le test.

Un test dure environ une heure, mais nécessite une heure supplémentaire pour le rinçage lorsque le sédiment est vaseux. L'ensemble du dispositif est transportable, de telle sorte que l'érodimètre peut être installé dans une camionnette ou sur un bateau (figure 2).

\subsection{Caractéristiques de l'instrument et formulation du flux}

Long de $1.20 \mathrm{~m}$, le canal, réalisé en polycarbonate, est transparent sur ses 4 faces. La veine d'écoulement a une section de $80 \mathrm{~mm}$ de large par $30 \mathrm{~mm}$ de hauteur. Cette dernière peut être réduite en ajustant un "faux plafond" au canal, de façon à 
augmenter la gamme de contraintes de cisaillement. De cette façon, les effets de bords sont réduits et dominés par les cisaillements près des parois inférieure et supérieure (l'épaisseur des couches limites correspondantes étant contrainte par la faible hauteur d'écoulement). Dans ces conditions, la longueur de $80 \mathrm{~cm}$ qui précède le sédiment à éroder est suffisante pour que le régime d'écoulement et la couche limite de fond (d'épaisseur centimétrique) soient établis. En outre, un abrasif de rugosité moyenne a été collé sur le fond du canal pour accentuer les turbulences et atténuer un éventuel effet de contraste lors de la transition entre le fond du canal et le sédiment naturel.

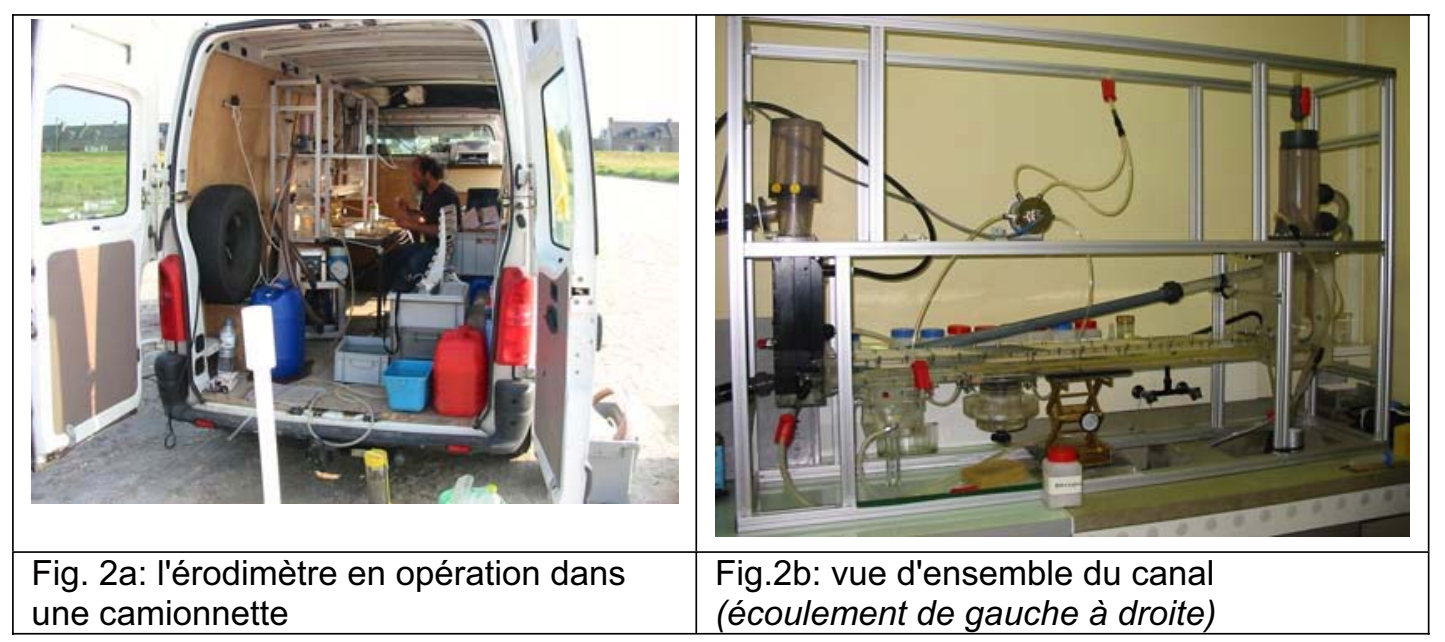

Le diamètre interne des carottes est de $9 \mathrm{~cm}$, correspondant au diamètre du disque de sédiment à éroder, tronqué par la largeur intérieure du canal de $8 \mathrm{~cm}$. Considérant que statistiquement l'érosion se fait sur une surface moyenne $\mathrm{S}$ de 6 $\mathrm{cm} \times 5 \mathrm{~cm}$, le flux d'érosion est déduit de la variation de turbidité $\mathrm{C}$ mesurée, affectée au volume total du canal, soit environ $\mathrm{V}=181$. On écrit finalement:

$$
\mathrm{E}\left(\mathrm{kg} \cdot \mathrm{m}^{-2} \cdot \mathrm{s}^{-1}\right)=(\mathrm{V} / \mathrm{S}) \mathrm{dC} / \mathrm{dt}=\left(18 \cdot 10^{-3} / 30 \cdot 10^{-4}\right) \Delta \mathrm{C}=6 \Delta \mathrm{C}\left(\mathrm{en} \mathrm{kg} \cdot \mathrm{m}^{-3}\right)
$$

$\Delta \mathrm{C}$ représentant l'augmentation de concentration chaque seconde. La valeur de $\mathrm{E}$ est moyennée sur 1 minute environ pour filtrer les fluctuations de la mesure de turbidité.

\subsection{Calibration et validation}

La calibration du dispositif en contraintes de cisaillement, et le contrôle de l'uniformité de ces contraintes sur l'échantillon de sédiment, ont été réalisés en testant le début de remaniement de matériaux non cohésifs.

L'uniformité des contraintes a été évaluée en examinant la distribution spatiale du début de remaniement d'un échantillon de sable bien trié, préalablement coloré par bandes perpendiculaires à l'écoulement (Fig.3). 


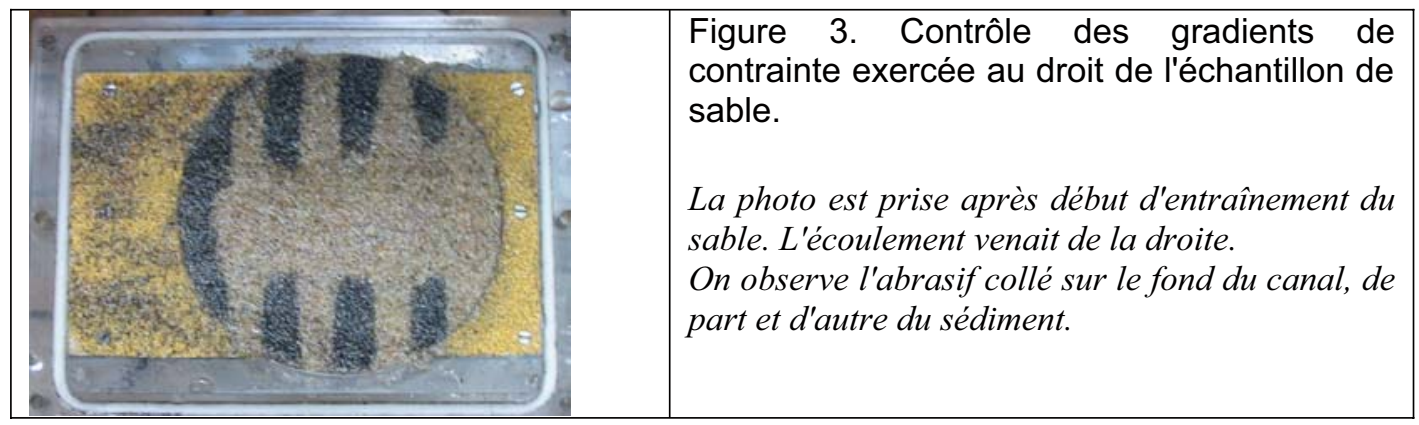

Ce type de test extrêmement sensible a révélé des différences significatives entre l'axe du chenal et les bords, qui ont été largement atténuées en ajoutant une rugosité centrale (des "Lego") sur le plafond du canal. Après essais sur de multiples configurations, un compromis acceptable a pu être trouvé.

La calibration en contraintes a été obtenue en recherchant le débit critique $\mathrm{Q}$ dans le canal à partir duquel un matériau d'une granulométrie et d'une densité donnée commence à bouger "significativement". L'application du critère de mise en mouvement de Shields permet d'en déduire la contrainte de cisaillement appliquée. On dispose ainsi d'une relation entre le débit imposé et la contrainte de cisaillement. Cette relation dépend normalement de la rugosité du sédiment, mais on suppose que sur les fonds de vase naturels l'écoulement reste turbulent lisse.

La calibration s'écrit:

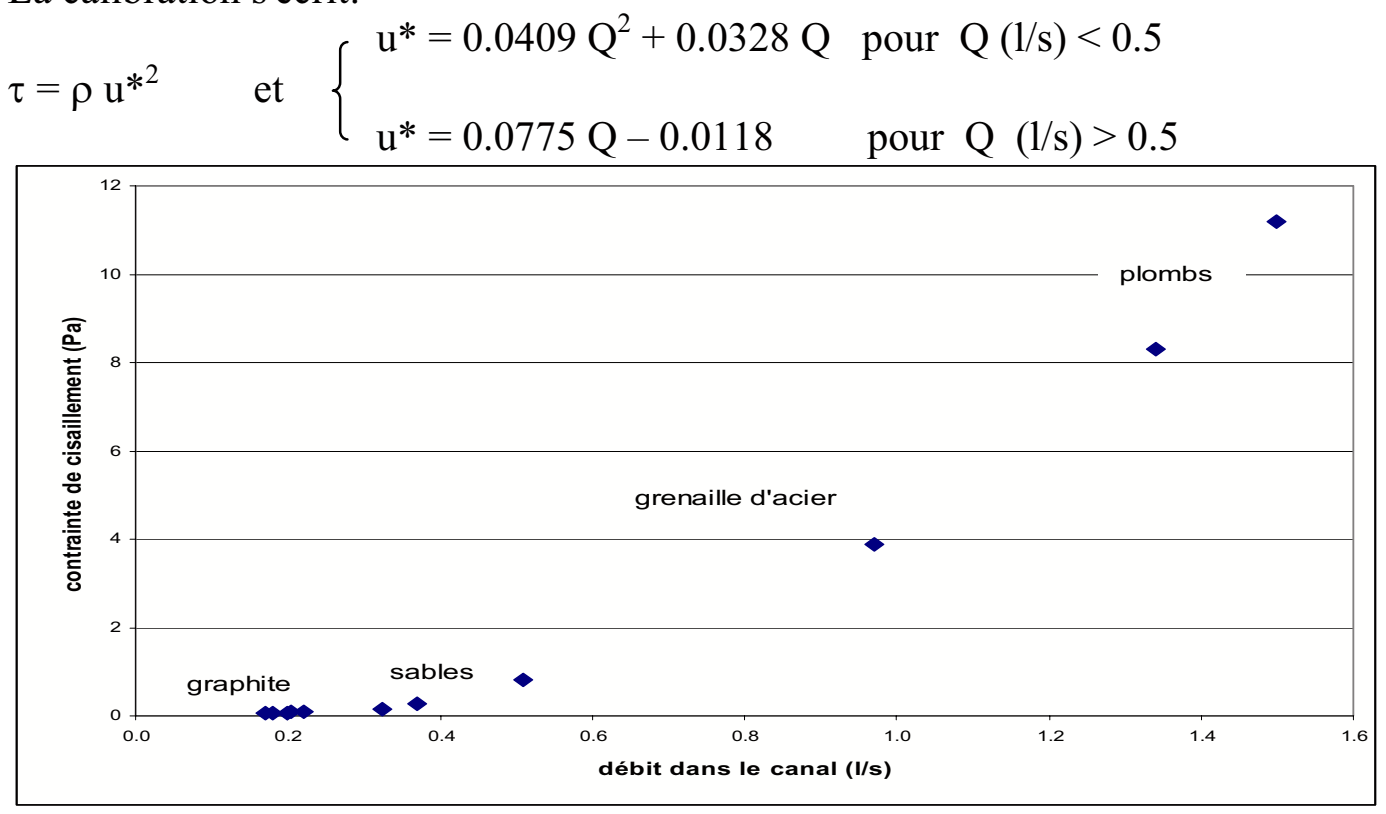

Fig. 4 : calibration de l'érodimètre en contrainte

\section{$\underline{\text { 3. Exemples de résultats }}$}

L'instrument a été déployé sur le terrain, aussi bien dans une camionnette stationnée au bord de l'estran que sur un bateau, permettant des tests sur des 
sédiments naturels purement vaseux (fig. 5) ou mixtes (fig. 6). Mais il s'est avéré très précieux en laboratoire pour l'étude du comportement de mixtures préparées.

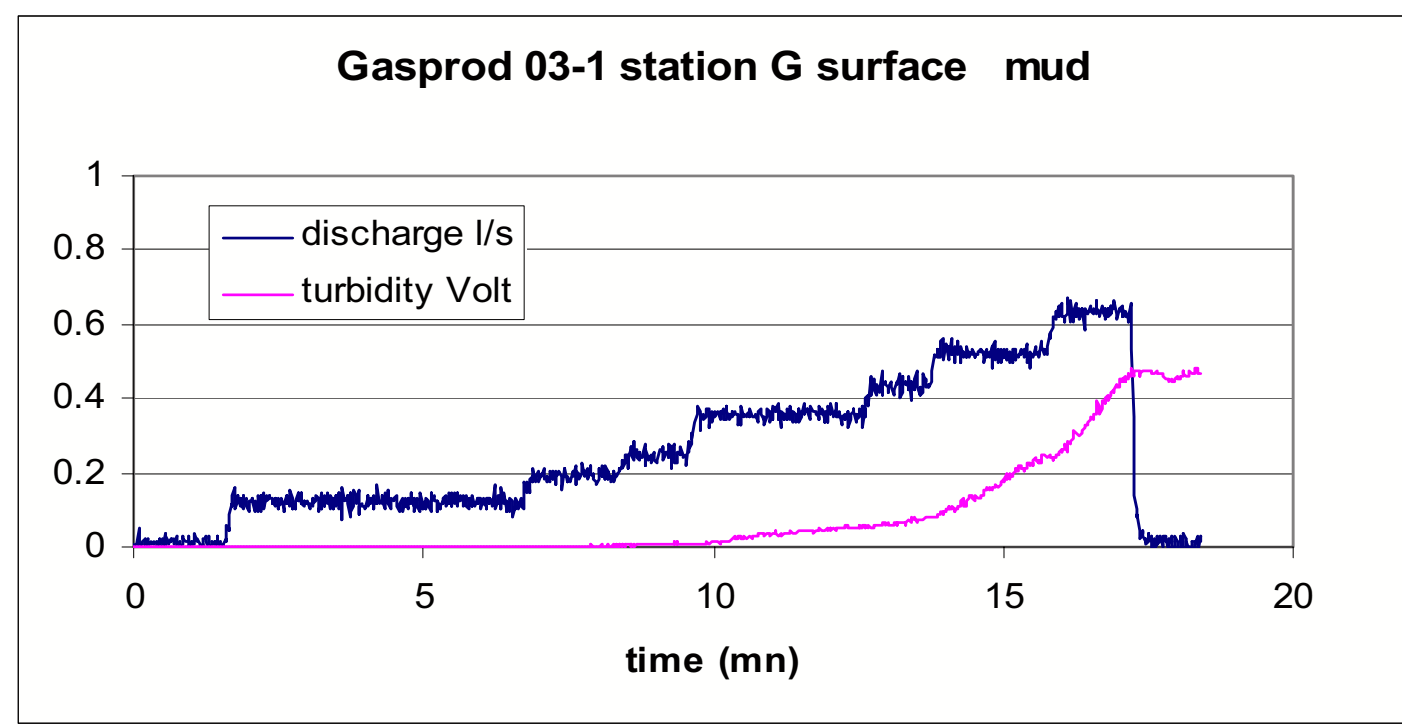

Fig. 5 : exemple de résultats bruts d'un test d'érodimétrie à bord de la Thalassa sur une vase naturelle du secteur Sud-Glénans, prélevée par $90 \mathrm{~m}$ d'eau

Ainsi, l'augmentation de la contrainte critique d'érosion d'un mélange sablovaseux avec la fraction volumique de vase a été mise en évidence pour plusieurs types de sédiment (Le Hir et al., soumis) : l'augmentation est progressive et linéaire si le sable est fin, ou au contraire présente un seuil en dessous duquel le sédiment reste non cohésif si le sable est de granulométrie moyenne.

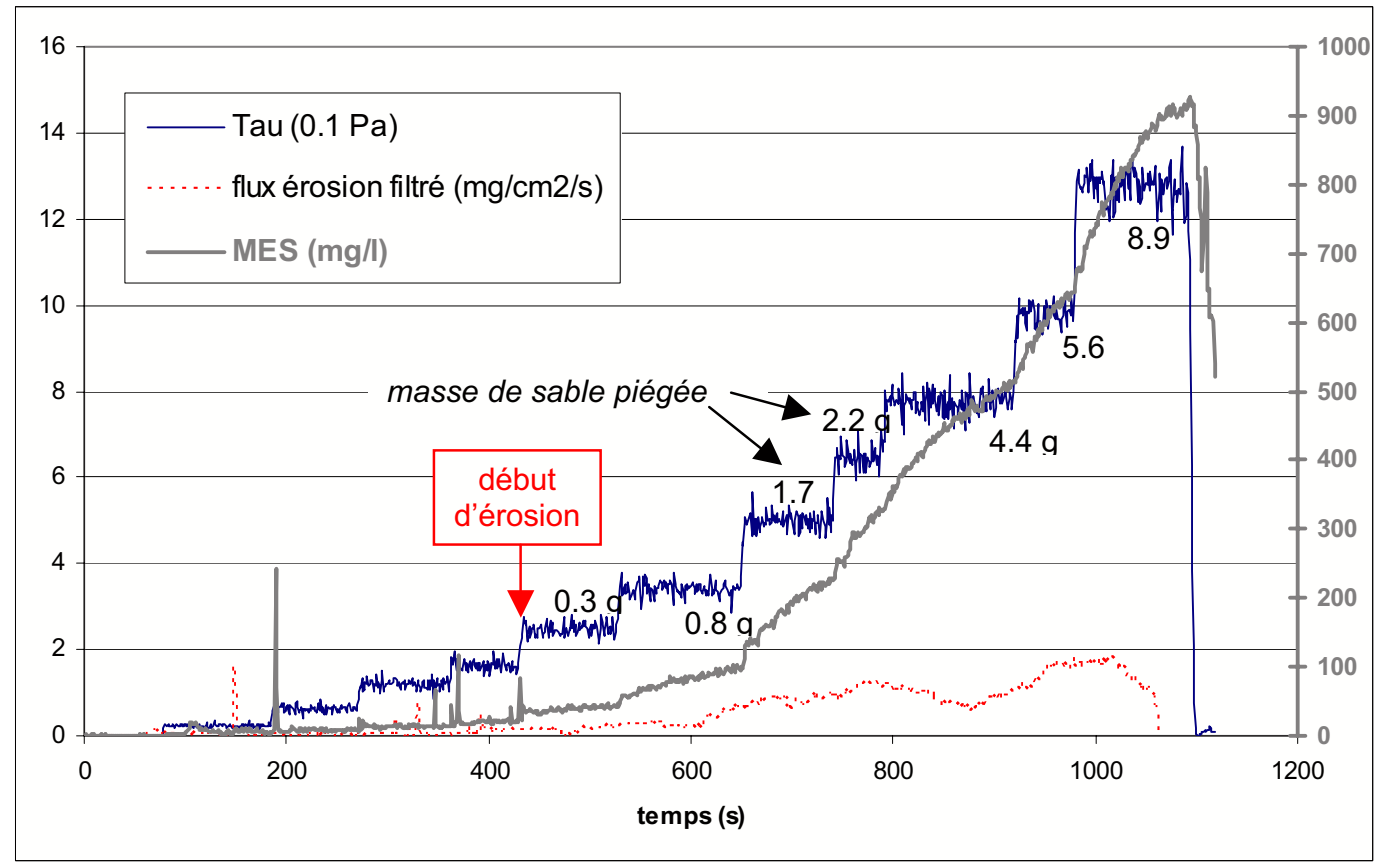

Fig. 6: Résultat d'un test érodimétrique sur un échantillon naturel prélevé dans l'estuaire de la Penzé (Finistère): mélange de sable fin et de vase ( $47 \%$ en volume). 


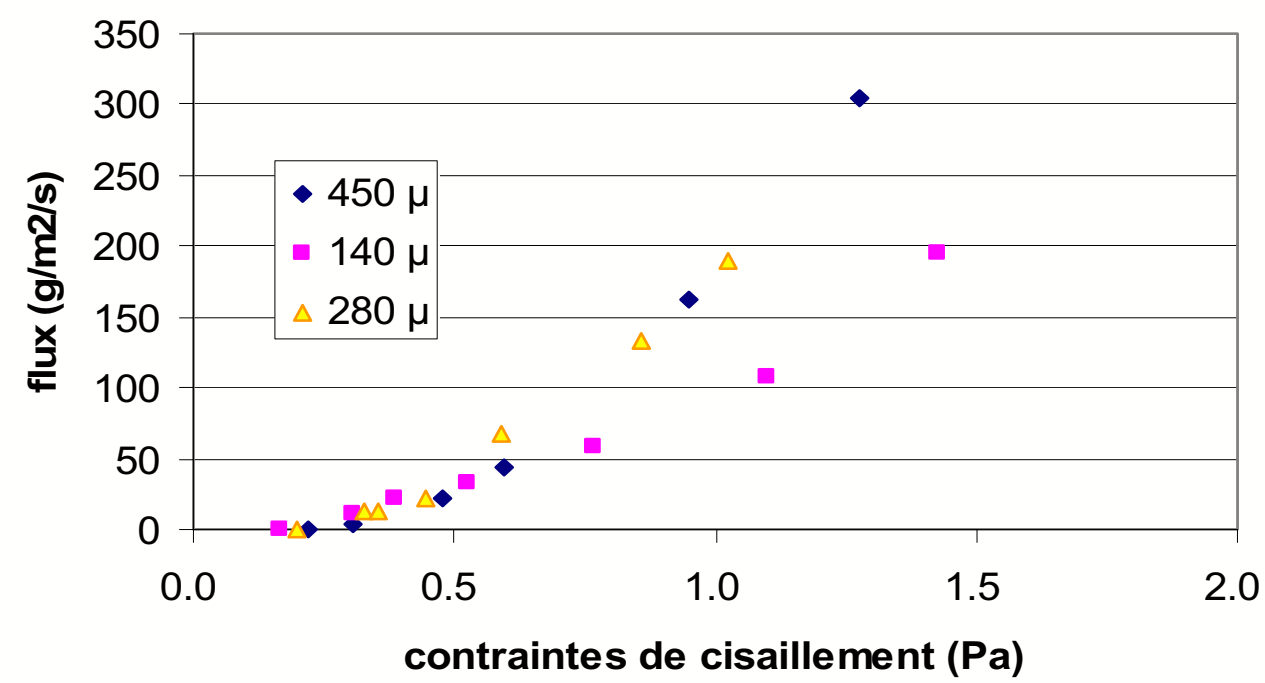

Figure 7 : mesure du flux d'érosion de sables fins et moyens

Des tests sur des échantillons de sable trié (fig.7) ont également permis de déterminer des flux d'érosion de sédiments fins non cohésifs. De plus en plus ces sédiments, dont les modes de transport dominants sont la suspension ou la saltation, sont modélisés par résolution d'une équation d'advection/diffusion. Le flux d'érosion constitue alors un terme source auquel les lois de capacités de transport classiques ne donnent pas accès. La figure 7 montre que le flux d'érosion varie peu en fonction du diamètre dans la gamme des sables moyens.

\section{$\underline{\text { 4. Discussion }}$}

L'expérience acquise au cours des tests effectués et lors de l'élaboration du protocole expérimental soulève de nombreuses questions utiles à la modélisation.

Tout d'abord la méthode de calibration de la contrainte de cisaillement est dépendante de l'estimation (arbitraire) du début d'entraînement des sédiments cohésifs et mérite d'être confrontée à une mesure précise de contrainte par vélocimétrie laser ou PIV. Une correction de rugosité devrait aussi être appliquée à la contrainte critique mesurée dans le cas du fond de sphères de plomb (contrainte de $12 \mathrm{~Pa}$, fig. 4). Mais la question de l'uniformité de la contrainte sur un fond naturel rarement lisse, et fréquemment recouvert d'organismes "saillants" reste entière. Dans le cas où les rugosités sont macroscopiques, la contrainte moyenne est-elle le forçage adéquat, représentatif des cisaillements locaux ?

Les formes d'érosion sont rarement uniformes (mais jamais au même endroit, ce qui ne révèle pas de biais opératoire), probablement comme dans la réalité: devrait-on cependant appliquer l'érosion à toute la surface du sédiment lors du calcul du flux, ou à une fraction seulement, estimée statistiquement ? D'autre part les flux d'érosion sont rarement constants au cours des paliers de courant, et il n'est pas toujours possible d'évoquer une stratification du sédiment pour expliquer 
leur décroissance: ce phénomène, qui n'est pas systématique, reste encore à préciser. Lors de l'échantillonnage sur estran, l'hétérogénéité spatiale du sédiment à petite échelle a souvent été observée : un traitement statistique des flux d'érosion est envisagé, rendu possible par la courte durée des tests. La démarche sera aussi facilitée en recherchant des corrélations avec des mesures rhéologiques simples d'accompagnement (par exemple avec un scissomètre de surface, Bassoullet et Le Hir, 2006)

Enfin, la pertinence du seuil d'érosion est discutable. En effet, il est souvent difficile à identifier, le flux d'érosion étant alors très faible et bruité. Lorsque c'est le cas, des lois d'érosion moins sensibles au seuil ("défaut" caractéristique de la loi de Partheniades) sont à rechercher.

\section{$\underline{\underline{5 . C o n c l u s i o n}}$}

L'érodimètre présenté permet de mesurer, après très peu de remaniement, l'érodabilité des sédiments fins naturels, souvent constitués de mélanges sablovaseux. Il est associé à un moyen de prélèvement en subtidal ou en intertidal. Son intérêt principal est sa portabilité et la relative rapidité des tests. Il est cependant limité par la faible rugosité (millimétrique) à ne pas dépasser, ce qui empêche d'étudier l'effet des macrophytes (mais pas celui du microphytobenthos) ou de nombreuses espèces de macrozoobenthos. Enfin l'action spécifique des vagues (en particulier la liquéfaction par les gradients de pression) ne peut être considérée.

\section{Références}

Bassoullet P., Le Hir P., 2006. In situ measurements of surficial mud strength: a new vane tester fitted to soft intertidal muds. Cont. Shelf Res., Bioflow special issue (sous presse).

Black K. and Paterson D.M., 1997. Measurement of the erosion potential of cohesive marine sediment: a review of current in situ technology. J Mar Environ Eng 4: 43-83

Migniot C., 1989. Tassement et rhéologie des vases, 2ème partie. La Houille Blanche, $\mathrm{N}^{\circ} 2-1989,95-111$.

Le Hir P., Cann P., Waeles B., Jestin H. \& Bassoullet P., soumis. Erodability of natural sediments: experiments on sand/mud mixtures from laboratory and field erosion tests. Proceedings of Intercoh'05, 20-23 Sept. 2005, Saga (J).(Soumis)

Paillard M., Morel J.-P. et Le Hir P., 1998. Projet "érodimètre": étude de faisabilité d'un dispositif de mesure de l'érodabilité des sédiments cohésifs. Rapport Ifremer DITI/GO/MSG 98.192, 105 P. + annexes.

Tolhurst, T.J., Black, K.S., Paterson, D.M., Mitchener, H.J., Termaat, G.R., Shayler,S.A., 2000. A comparison and measurement standardisation of four in situ devices for determining the erosion shear stress of intertidal sediments. Continental Shelf Research, 20, 1937-1418. 